\title{
Design of AP Network Subsystem Based on WLAN System
}

\author{
Yang Zhuoran, Zhang Yanmei \\ School of Information Engineering, China University of Geosciences (Beijing), Beijing, China
}

Keywords: AP, Network Subsystem, WLAN.

\begin{abstract}
In recent years, the rapid development of wireless communication technology, especially with wireless LAN technology is widely used in notebook computers, smart phones and tablet PCs and other Wi-Fi terminal equipment, more and more families, institutions and enterprises began to use a WLAN large-scale wireless network. Wireless Internet access can bring great convenience to people's lives and work, and with the wireless networking complexity and expanding scale, use a lot of AP can not be avoided, which requires the use of more convenient and more effective methods for controlling and AP management, while providing a good interactive WEB network management interfaces for network administrators.
\end{abstract}

\section{Introduction}

Historical development of wireless communication technology can be traced back to 1896 when the Italian Guglielmo Marconi invented the telegraph, after a hundred years of the development of wireless communications technology has made great strides. With the rapid development of Internet communications, networks change from traditional cabling network development to the wireless network. With the gradual mature Wi-Fi technology, on the market a variety of Wi-Fi terminal such as laptops, wireless PDA, support for Wi-Fi enabled mobile phone that supports Wi-Fi enabled consoles and supports $\mathrm{Wi}-\mathrm{Fi}$ enabled digital cameras sprung up, prices are getting lower and lower. Compared with several other wireless communication technologies currently, Wi-Fi technology advantage is obvious, and $2 \mathrm{G} / 3 \mathrm{G}$ compared, Wi-Fi is 10 times the bandwidth of $3 \mathrm{G}$, while distribution network costs are much cheaper than $3 \mathrm{G}$. Wi-Fi as a wireless connection protocol, it is a major way and the way to achieve WLAN C Wireless Local Area Networks) are, WLAN that is, wireless local area networks, is a use of RF (Radio Frequency) technology for data transmission systems, the use of network coverage of wireless communications technology in a certain space range established wireless communication technology and computer network technology product of the combination, it wireless multiple-access channel as a transmission medium, can provide a traditional wired LAN (Local Area Network) feature, It enables users to realize the true anytime, anywhere, free broadband Internet access ${ }^{[1]}$. The emergence of the technology to enrich the communication transmission type, make up the traditional wired local area network deficiencies, achieve the purpose of extending the network so that wireless local area network can be accessed using a simple architecture that allows users to achieve no net line limitation through it unobstructed access to the network. Daily learning to meet the people that work and life is playing an increasingly important role. 


\section{Wireless local area network technology}

IEEE 802.11 WLAN protocol is the IEEE 802 standard IEEE Institute established by the Committee, the standard defines a wireless LAN physical layer (PHY) and media access control layer (MAC) protocol specification that allows wireless LAN and wireless equipment manufacturers to establish within a certain range interoperability of wireless networks.

802.11 networks most basic kernel module is a basic service set (BSS), it is only by the workstation group composed communicate with each other. Communication occurs within a gray zone, the area is called the basic service area, it is by the propagation characteristics of the wireless medium to decide. When a mobile station within the basic service area, it can and BSS other members chant letter. BSS there are two different structures:
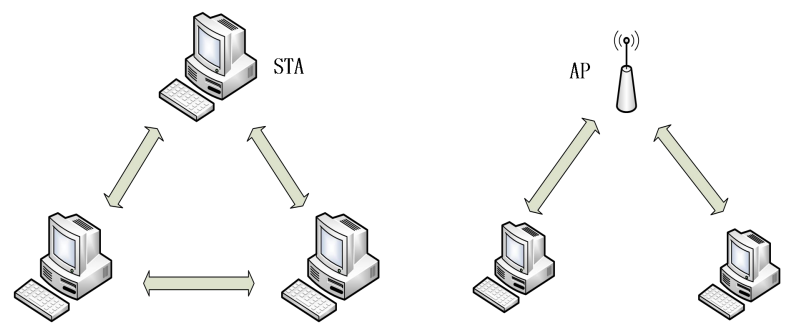

Figure 1. BSS structure

In an IBSS, direct communication between mobile stations, so they must be within the effective range of their communications. IBSS may be only two mobile stations, which is the smallest possible 802.11 network situation. IBSS is usually used between the mobile stations to establish a short communication, when the communication ends, IBSS also disbanded. Because, they are of short duration, IBSS sometimes is called as Ad-hoc BSS. It uses radio frequency technology (Radio Frequency) data communication, radio wave is transmitted media data, it is not necessary in order to replace the old wires and cables requires a lot of wiring inconvenient wired LAN constituted by a twisted pair. But in the current wireless LAN system, it will still be wired backbone network cable. Wireless local area network system has its own access infrastructure, wireless signal coverage in a user via one or more wireless access points to join the current wireless local area network ${ }^{[2]}$. Now in public areas and a large business district, university campuses, airports, etc., the wireless local area networks have been widely used.

\section{Subnetting IP addresses}

IP is a network interconnection agreement between the network transport protocols IPv4 and IPv6 network layer contains the sub-version, is a host in the Internet a way of addressing. We know each other communicate between different phone through different phone numbers to achieve, also in computer networks in order to achieve different network computers and other devices communicate with each other also need to assign them an access network dedicated number this number is the IP address. In Internet communications, between different hosts by IP address information transmission and switching, IP protocol is to realize this function. IPv4 is a connectionless protocol, will make every effort to deliver a packet, the packet can not be one hundred per cent guarantee sent to the destination, we can not absolutely guarantee that all packets are sent one by one in the correct order to reach the goal. These aspects are processed by the upper layer transport protocol, such as Transmission Control Protocol. IPv4 address consists of four segments 32, of which eight each, a total of 4,294,967,296 addresses. Usually easy to write and remember that we do not write 32-bit binary form, but each of the IPv4 address uses 4 segments in dotted decimal notation, each numeric value ranges from 0 to 255 , between adjacent segments divided by the number of points, shaped like 
123.123.123.123. And this was 32 by the network number and host number two parts, respectively, the network and host addresses. In order to meet the practical application of network scale size of the points, IP address, according to the designers of the network number and host number of bits occupied by different grouping of IPv4 addresses, a total of A, B, C, D, E five, network range from big to small. Restricted IPv4 address plus the length of the recent decades of rapid development of Internet technology, the number of IPv4 addresses have access to the network can not meet the growing demand for the number of hosts. And it has a large number of IP addresses next-generation Internet protocol IPv6 IP can be a good solution to this problem thus completing the Internet network layer packet switching. IPv6 Internet Protocol version 6 is the English name, meaning the sixth version of Internet protocol. IPV 6 address by the $\mathrm{g}$ section $12 \mathrm{~g}$ of the composition, each have their own 16 with four hexadecimal number indicates the range of numbers is OOOO FFFF, each separated by a colon, so the full IPv6 address should have 32 digit hexadecimal number, shaped like: 1234: 2345: 3456 : 4567: ABCD: ABEF: 12BC: FFFF, is a complete and valid IPv6 address. As shown in Figure 2.

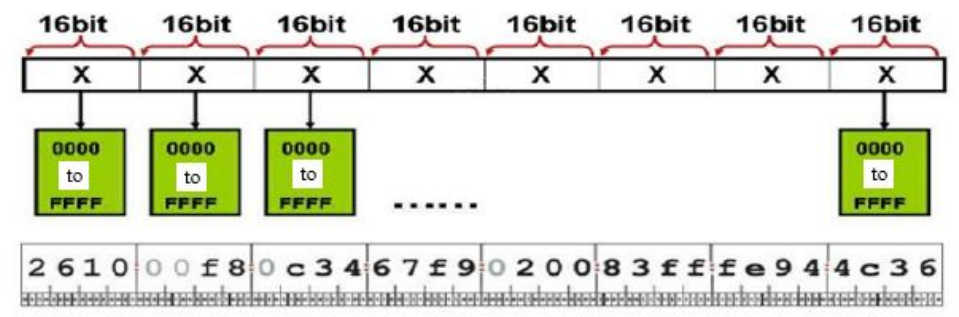

Figure 2. IPv6 address representation

IPv6 unicast address can be used to identify a network interface. According to the relevant protocol rules packets cast gave its interface according to the corresponding address. IPv6 unicast addresses have a category name indicates special address, such as the link-local address and a unique area address. As in IPv6-specific data transmission, anycast role is to provide support to service-oriented IP address; it can also be assigned to multiple nodes to provide a specific service the same address, similar to a combination of the IPv4 unicast and multicast. Like multicasting, as a group of receivers address entries for the node, when a packet is designated as anycast, the only transferred to the nearest or the routing table to determine the lowest cost of a receiving address, the receiver address respond after receiving the data packet, and then the subsequent transmission. The IPv6 multicast address can identify multiple interfaces to a multicast address to send data packets can be sent to this address to identify them on all interfaces. Multicast address starting byte all "1", i.e. FF00 :: / 8. The second byte before 4 When set to 0 , said permanent multicast address, when set to 1 , indicating a temporary multicast address. Multicast addresses can not be used as a routing header in the middle of the target and source addresses.

\section{Design AP network subsystems}

In our real life, due to the location and the surrounding environment, it can not be fully achieved complete access to wired communications, but for convenience and quick consideration, in many places we tend to use the wireless network coverage approach. Such as shopping malls, collective office business district, university campus and so need to place a wide range of wireless network coverage. To achieve a wide range of wireless network coverage, they often need to set up a large number of AP in different geographical locations to meet the needs of high coverage of the wireless network. AP distributed in so many different places, it relies on traditional management methods difficult to make AC effective unified management ${ }^{[4]}$. It is because of the need to facilitate and efficient management so much AP; we need to establish a new large-scale management of the AP program. We propose is based on the IP subnet to AP group to group management. 


\subsection{ARP packets based on the IP address of the network management module overall demand}

The project needs analysis using NMS able to AP by IP network packet functions with Configuration and management. Network management structure of the module of this system is shown in Figure 3. Command line module, WEB module, SNMP C Simple Network Management Protocol) module together constitute the NMS system, according to the specific circumstances and needs, this project need only implement the AP at the command line network management and network management WEB IP network by grouping capabilities for network management. Design and development in the NMS is an attempt to provide users with a command-line mode and two AP WEB mode network by IP packet-based network management. Wherein CMP C Control Management Plane) is flat configuration management plays a central role in the whole device system. Command line mode operating system, WEB and SNMP network management model management model is three different configuration management tools, plays the same functionality as in some cases, for getting and setting configuration message data, notably the completion of the appropriate configuration and management functions. Wherein, APP module in this project is the AP group module. The reason CFA module is configured proxy, the proxy configuration is introduced in order to support the implementation of a distributed configuration commands, to facilitate future expansion. The project needs at the command line network management module for the selected group configuration AP IPv4 and IPv6 network segment, and can get the current AP group configuration IP network through the corresponding command. AP pressing need to design IP network packets WEB interface and the corresponding spooler WEB network management module, the selected group display and set AP IPv4 and IPv6 network configuration are achieved. Including IPv4 address input text box, IPv4 subnet mask input text box, IPv6 address input text box, IPv6 prefix length of the input text boxes, and complete spooler this network management function. Configuration tool to configure the data parameters are message-based, between configuration messages and parameters to establish a correspondence between the configuration management plane can be configured via messages into standard configuration management objects, while allowing each configuration has a unique message ID.

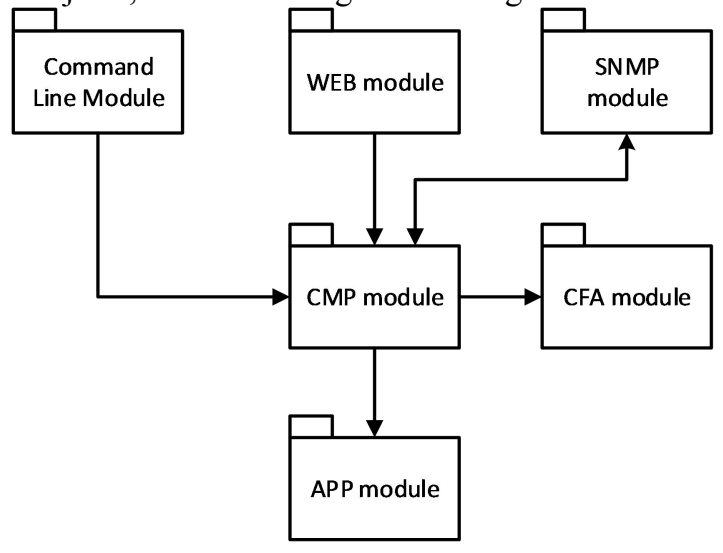

Figure 3. Network management structure

\section{2 AP grouped by IP network demand}

AP grouped by IP network can provide a method for unified management of the AP region according to the user. AP existing groupings in the AP name or type as the basis for grouping by the AP template to determine when added AP Group grouping AP. In particular, if the Auto AP (automatic AP) was added to a certain template, then use the template line Auto AP will be assigned to the group. Packets not explicitly specified configuration AP, PnP AP (Plug AP) and not previously added to the template's Auto AP is AP Group belongs default_Group. 
AP grouped by IP network is in the original AP grouping for the AP Group (currently required by the IP network packet AP group) to increase IP network configuration. When the AP on cable network its IP address with the AP group match at the configuration can be achieved by IP network packet AP effect. Specifically by the system administrator to complete the configuration of a network by IP packet AP group in the command line mode, including IPv4 and IPv6 configuration or configured separately situation. In the W EB NMS add the appropriate location in the packet management configuration WEB interface, to meet the system administrator via WEB NMS for selected AP group IP subnet configuration and management.

\section{Unit testing}

Unit testing also known as UT is the smallest unit of software modules for testing. Mainly from the local data structures within the module, it is important the execution path, unusual execution path and module interface four aspects to consider, the basic use of white box techniques. First, test the data structure, and then choose a different path to perform the test, but also on the wrong path and boundary value test, final test data stream through the module interface. Unit testing, since a single module can only complete part of the function, it is necessary to develop the drive module and support module for each module. Driver module, in the unit test module test module calls are to realize the role of the main program, while receiving additional test data is then passed under test modules. Support Module, also known as stubs, used in place of subordinate modules is tested in unit testing module.

The unit tests using the mirror test tools Mirror Test Tools, simultaneously with the python scripting language to set the parameters, variables, functions, and rollback functions pile like. The main function of the number of IPv4 and IPv6 network configuration tested when it comes to legitimacy, check IPv6 address and prefix length of validity check IPv4 address and network mask, according to the availability of IP address and network mask or prefix length corresponding network segment to determine whether the segment has been configured in accordance with pre-configured network, configuration conflicts inspection. Mirroring data and state testing tools will run under the real system environment mapping to a simulated environment, the formation of mirrors, the need for unit testing function is loaded into the simulated environment, and set the necessary environment variables, functions into the Senate, will function Replace the external interface to meet the expected results of the function call. For each test write different content UT use cases, execute scripts, use cases to achieve UT statement coverage of test functions, logical coverage, condition coverage, decision coverage, analysis of test results.

\section{Acknowledgement}

This paper is supported by National Natural Science Foundation of China (91328201).

\section{Reference}

1. Yang Rong.WLAN network design and application analysis [D]. Beijing: Beijing University of Posts and Telecommunications, 2006.

2.William Stallings. Data and Computer Communications [M]. Beijing: Higher Education Press, 2009:543-617.

3. Zhu Xiaoyan, Liang Shengbin. Research subnetting IP address-based algorithm [J]. Computer Knowledge and Technology, 2012, 8 (22) 5324-5326.

4. Huang Yong. WEB Design and Implementation of IPv6 network system [D]. Wuhan: Wuhan Research Institute of Posts and Telecommunications, 2011. 